\title{
New developments in neonatal screening
}

\author{
Kim Bartlett, Simon J Eaton, Morteza Pourfarzam
}

There have been significant advances in the methods available for the detection and investigation of individuals with inherited metabolic disorders. The burgeoning of molecular biology in recent years and the discovery of new classes of inherited metabolic disorders, such as inborn errors of fat oxidation, are well known. What is perhaps less well recognised, is that there have been comparable advances in analysis and laboratory automation. The combination of the development of generic analytical technology of great power and sophistication, and the discovery of new treatable metabolic diseases detectable in the newborn period, has, we believe, resulted in a sea change in neonatal screening.

Neonatal screening for metabolic diseases became feasible as a result of the pioneering work of Guthrie. He developed a simple, robust, and effective technique for the detection of elevated concentrations of phenylalanine in blood-the "Guthrie Test"which was used to detect phenylketonuria by means of a semi-quantitative microbiological bioassay. ${ }^{1}$ Screening for this disorder (PKU; phenylalanine hydroxylase deficiency; McKusick 261600) was subsequently implemented in the UK in $1969 .^{2}$ The specimens used are dried blood spots which are usually collected by means of a heel-prick on the sixth day of life, although later sampling, up to 14 days, can occur. This form of specimen collection has become almost universal. Quantitative assays specific for phenylalanine have been introduced by some centres, driven by the desire to introduce a degree of automation. ${ }^{34}$ An alternative approach is to use chromatographic methods, and this has been adopted by some centres. ${ }^{5}$ The proponents of this technology contend that other inherited disorders of amino acid metabolism can be detected and that improved prognosis may be achieved by early diagnosis. However, chromatographic methods are time consuming and difficult to automate, particularly in respect of the recognition of abnormal chromatograms. None the less, the concept of a generic technology capable of detecting several disorders by a single procedure is attractive, and single dimension paper and thin layer chromatography with ninhydrin staining has been used successfully for many years in some centres in the UK for the detection of PKU and other inherited disorders of amino acid metabolism.

The justification for PKU screening is that early detection of affected individuals and the introduction of treatment, a diet low in phenylalanine, avoids irreversible brain damage. ${ }^{67}$ Similarly, the measurement of TSH or thyroxine for the detection of congenital hypothyroidism was adopted throughout the UK in
$1981^{8}$ and is also universal. The dried blood spot, it turns out, is a surprisingly robust form of sample collection and a plethora of conditions have been proposed as candidates for neonatal screening, although the benefits are sometimes far from clear. ${ }^{9-13}$ The ability to detect genetic disease directly by means of molecular biological techniques suggests that, at least in principle, screening for all inherited conditions is possible. However, in most inborn errors a given phenotype is caused by a large number of different mutations and thus if direct mutational analysis is contemplated a multiplicity of probes is required. Clearly, novel previously unknown mutations contributing to a given pathological phenotype would be missed.

Since the introduction of the Guthrie test there has been a remarkable expansion in the known number and types of inherited metabolic disorders. Thus new classes of disorders such as inherited defects of mitochondrial $\beta$-oxidation, peroxisomal metabolism, and of the respiratory chain are now known. Furthermore, the early concept of an inborn error of metabolism presenting as a florid biochemical disorder detectable in the first days of life has been modified by the recognition of episodic, late presenting disease. The commonest of the $\beta$-oxidation disorders, medium chain acyl-CoA dehydrogenase deficiency (MCAD), presents most frequently with episodic hypoglycaemia which may be life threatening. These hypoglycaemic episodes can be precipitated by otherwise unremarkable intercurrent infections, or simply by prolonged fasting. Conventional investigations during periods of remission are generally uninformative, but characteristic abnormal acylcarnitines are detectable in blood. MCAD is readily treated by the simple dietary manipulation of the avoidance of fasting, although if a metabolic crisis secondary to stress should occur, more aggressive intervention such as the intravenous administration of glucose is necessary. ${ }^{14}$ Thus MCAD, which is at least as common as PKU, is easily and cheaply treated, and failure to recognise children with the disorder and institute timely and appropriate management can lead to brain damage or death. MCAD is one of several inborn errors of mitochondrial $\beta$-oxidation. ${ }^{15}$ Long chain 3-hydroxyacyl-CoA dehydrogenase deficiency (LCHAD) and very long chain acyl-CoA dehydrogenase deficiency (VLCAD) are also members of this group of disorders and seem to be relatively common and amenable to dietary treatment. The question arises: is it possible to detect MCAD and other disorders of $\beta$-oxidation by analysis of dried blood spot specimens? 
Table 1 Inborn errors of metabolism detectable by Tandem mass spectrometric analysis of acryl-carnitines and amino acids

\begin{tabular}{lll}
\hline Inherited enzyme defect & $\begin{array}{l}\text { McKusick } \\
\text { number }\end{array}$ & Incidence \\
\hline Short chain acyl-CoA dehydrogenase & 201470 & $?$ \\
Medium chain acyl-CoA dehydrogenase & 201450 & $1: 8000$ \\
Very long chain acyl-CoA dehydrogenase & 201475 & $?$ \\
Long chain 3-hydroxyacyl-CoA dehydrogenase & 143450 & $?$ \\
Long chain-2,4-dienoyl-CoA reductase & 222745 & $?$ \\
Glutaryl-CoA dehydrogenase & 231670 & $1: 30000$ \\
Multiple acyl-CoA dehydrogenase & 305950 & $?$ \\
Propionyl-CoA carboxylase & 232050 & $1: 50000$ \\
Methylmalonyl-CoA mutase & 251100 & $1: 48000$ \\
Isovaleryl-CoA dehydrogenase & 243500 & $1: 50000$ \\
Branched chain ketoacid dehydrogenase & 248600 & $1: 185000$ \\
Phenylalanine hydroxylase & 261600 & $1: 8000$ \\
Tyrosinaemia type I & 276700 & $1: 100000$ \\
Non-ketotic hyperglycinaemia & 238300 & $1: 55000$ \\
3-Hydroxy-3-methylglutaryl-CoA lyase & 246450 & $?$ \\
Citrullinaemia & 215700 & $1: 250000$ \\
3-ketothiolase & 203750 & $?$ \\
3-Methylcrotonyl-CoA carboxylase & 210200 & $?$ \\
Maple syrup urine disease & 248600 & $1: 290000$ \\
Camitine palmitoyltransferase I & 600528 & $?$ \\
Carnitine palmitoyltransferase II & 600650 & $?$ \\
\hline
\end{tabular}
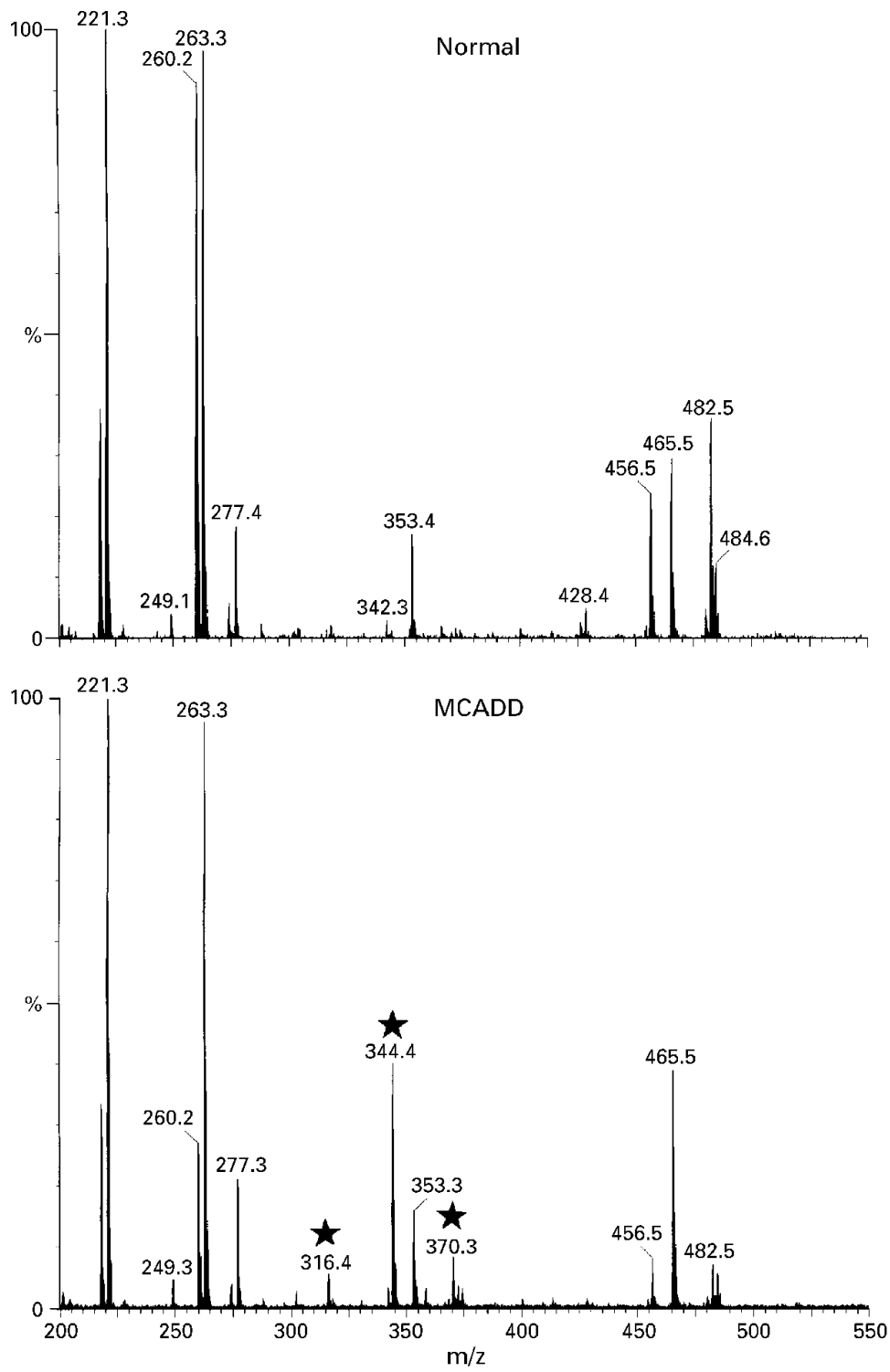

Figure 1 Tandem mass spectra (parents of $m / z 85$ amu; acylcarnitines) of butylated dried blood spot extracts from a control and an MCAD deficient subject (MCADD): * significant diagnostic analytes characteristic of $M C A D D$.
An inherited metabolic disorder may be identified by detection of an abnormal gene, an abnormal gene product, an abnormal gene product property (enzyme activity) or the presence of an abnormal metabolise resulting from attenuation of a metabolic pathway. Most techniques currently in use rely on the detection of abnormal metabolises usually by means of some form of chromatography. Thus analyses of blood and urinary amino acids and urinary organic acids are commonly used for the investigation of children who present with symptoms. Chromatographic methods are time consuming, difficult to automate and, although not an issue for the investigation of the relatively small numbers of patients presenting in hospital, for the purpose of neonatal screening, are inappropriate. A generic technology capable of detecting several disorders (including PKU and MCAD) but avoiding the disadvantages of chromatography is clearly preferable to a large number of single disease tests. Tandem mass spectrometric (TMS) analysis of acylcarnitines and amino acids has been described recently ${ }^{16}{ }^{17}$ and pilot studies suggest that this technology is suitable for neonatal screening programmes.

Mass spectrometry is a powerful technique for the characterisation of the structure and of the molecular weight of compounds and for quantitative measurements and has found widespread biomedical applications. Several hyphenated techniques, whereby a mass spectrometer is linked to other chromatographic separation procedure, have been developed, of which gas chromatography-mass spectrometry (GC-MS) is perhaps the best known. Indeed, GC-MS is currently the method of choice for the analysis of urinary organic acids. In recent years tandem mass spectrometry (TMS) has become available for routine analysis. TMS offers major advantages over single stage mass spectrometry in term of speed and selectivity of analysis and specificity in molecular structure determination. In TMS two consecutive stages of mass analysis are used. The first stage of mass analysis separates a compound of interest from other parent ions produced in the ionisation source. Following this mass filtering stage there is a collision cell where the relatively stable molecular ions are caused to fragment, usually by collision induced dissociation (CID) with a neutral gas such as argon. The fragment ions produced are transmitted into the second mass analyser where they are separated and detected. The result is a "daughter" or "product" ion spectrum of the selected precursor essentially free of interferences from other components present in the sample which can be interpreted to reveal structural information of the selected compound. Other common TMS experiments include precursor ion scan and neutral ion scan.

One of the major applications of TMS is the analysis of mixtures by eliminating the need for chromatographic separation or purification of individual components of complex samples (such as dried blood spots), because separation and analysis take place simultaneously and entirely within the mass spectrometer. In 

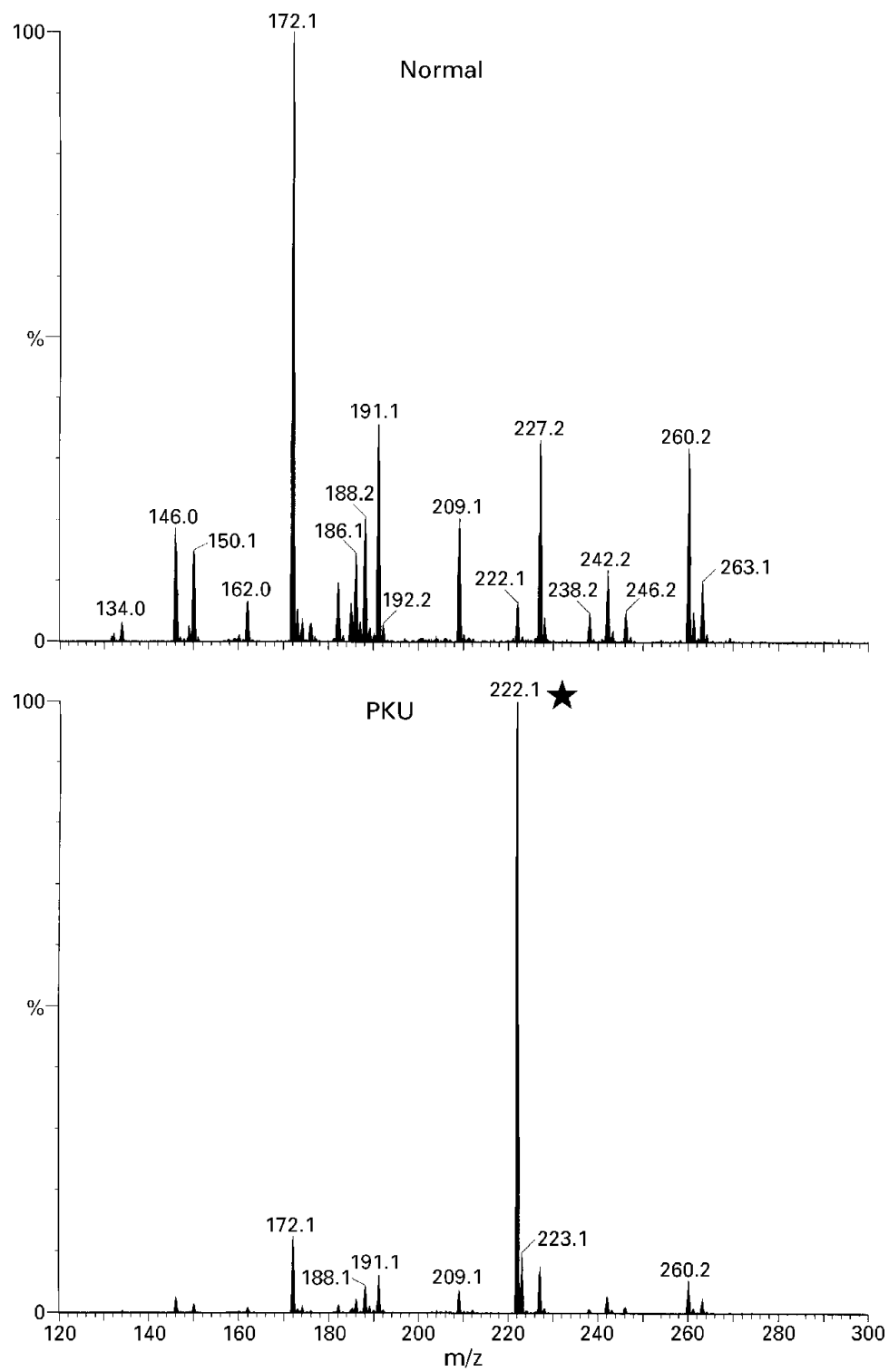

Figure 2 Tandem mass spectra (neutral loss of m/z 102 amu; amino acids) of butylated dried blood spot extracts from a control and a child with PKU: ${ }^{\star}$ significant diagnostic analytes characteristic of PKU.

the context of acylcarnitine analysis all acylcarnitines undergoing CID produce a common daughter ion $(\mathrm{m} / \mathrm{z}=85 \mathrm{amu})$ and therefore if MS1 is scanned and MS2 set to detect only ions of mass $85 \mathrm{amu}$ ("parents of 85 scan"), an instantaneous analysis of acylcarnitines is achieved. A similar approach can be used for the detection and quantitation of amino acids and thus as a sample is introduced into the source of the instrument two analyte specific scan functions are used to detect first acylcarnitines and then amino acids. Some typical examples are shown in figs 1 and 2 taken from our own studies. In principle, further scan functions can be designed to detect further classes of analyses such as bile acids and therefore other classes of disorders can be detected. In theory, then, any disorder in which the substrate of the defective enzyme is a CoA ester will result in the formation of the corresponding carnitine ester and such a defect is detectable by acylcarnitine analysis. Clearly, any disorders characterised by accumulation of the parent amino acid will become apparent by amino acid analysis. Table 1 summarises the disorders which are detectable by means of TMS analysis of acylcarnitine and amino acid analysis of dried blood spots. It should be noted, however that the incidences quoted are citations and are population dependent.

Recently, a combined retrospective and prospective pilot screening programme was reported at the 1996 meeting of the International Society for Neonatal Screening (Naylor EW, Expanded screening for metabolic disorders; abstract) In brief, a total of about 27000 blood spots were analysed by two centres (Pittsburgh and Rayleigh, USA). The total number of positive results detected was 69 , giving an overall incidence of 1 in 3900 , and it is particularly noteworthy that of the total of 69 positives detected, 35 were PKU plus MCAD. The incidence of the other rarer disorders is probably inflated because the population screened included the Mennonite communities of Lancaster County, Pennsylvania, who have a particularly high prevalence of inborn errors. None the less the authors of this study claimed zero false positives and zero false negatives. Our own experience confirms this. We conducted a blinded multicentre study in which dried blood spots from babies with confirmed disorders, and from babies with no evidence of metabolic disease, were submitted to our laboratory for analysis by TMS. In all cases where an abnormality was anticipated it was found. There were no false positive results. ${ }^{18}$ TMS screening of dried blood spots is therefore a technique of high sensitivity and specificity. It might be suspected that a complex analytical technology such as TMS would be inappropriate in the context of routine service delivery. However, our experience is that the method is robust and should be seriously considered for pilot screening programmes in the UK.

What, then, is the cost of a neonatal screening programme based on this technology? The simple analytical cost of TMS is undoubtedly higher than current technologies, but the cost per test will depend on the total number screened because the major fixed cost is the amortisation of instrument purchase. Set against this, however, are some major cost benefits:

(i) the recall and retest rates for analyses carried out by means of TMS are close to zero;

(ii) the technique is quantitative and thus follow up by means of conventional (and expensive) amino acid analysis is unnecessary;

(iii) management of positive cases and monitoring of dietary control can be achieved by the same technology;

(iv) it is highly probable that other disorders are amenable to concurrent analysis of the same blood spot with the added value of one stop testing;

(v) the adoption of TMS screening, as it is inherently more sensitive, allows neonatal 
screening to be carried out earlier than the conventional 6-14 days of life, and earlier treatment will result in improved outcomes.

The costs relating to sample collection, administration, and follow up would be incurred irrespective of the analytical method used. However, it is obvious that the effective detection of an increased number of patients with inherited metabolic disease, most of whom are treatable, in the early neonatal period, will place an additional burden on clinical services. It could be argued that the early detection and treatment of such infants will result in savings to society in that lifelong disability is avoided. Furthermore, even if the prognosis is poor, as it is for some of the rarer conditions, effective screening will at the very least provide parents with genetic advice early in their reproductive life. But this does not mean that there will be savings to the health service-most probably the reverse. Thus the body charged with determining how to spend its limited budget, the NHS, has a vested interest in promoting policies which do not result in further financial burdens. Neonatal screening is one of many medical interventions currently the subject of meta-analysis commissioned by the NHS, in the "Health Technology Assessment" exercise. We await the results with great interest.

1 Guthrie R, Susi A. A simple phenylalanine method for detecting phenylketonuria in large populations of newborn infants. Paediatrics 1963;32:338-43.

2 Department of Health and Social Security, Welsh Office. Screening for early detection of phenylketonuria. HM(69) 72 1969. Cardiff: DHSS

3 McCaman MW, Robins E. Fluorometric method for the determination of phenylalanine in serum. 7 Lab Clin Med 1960;55:885-90.
4 Kirkman HN, Carroll CL, Moore EG, Matheson MS. Fifteen year experience with screening for phenylketonuria with an automatic fluorometric method. Am 7 Hum Genet 1986;34:743-52.

5 Ireland JT, Read RA. A TLC method for use in neonatal screening to detect excess aminoacidaemia. Ann Clin Biochem 1972;9:129-32.

6 Snyderman SE. Newborn metabolic screening: follow-up and treatment results. In: Carter TP, Wiley AM, eds. Genetic disease: screening and management. New York: Alan Genetic disease: screening an
R. Liss, 1986: 195-209.

7 Guthrie R. Newborn screening: past, present and future. In: Carter TP,Wiley AM, eds. Genetic disease: screening and management. New York: Alan R Liss, 1986: 319-39.

$8 \mathrm{DoH}$. Joint Standing Committee on Screening in Medical Care. Screening for congenital hypothyroidism. HN(81)20 and Appendix 1981. London: Department of Health and Social Security.

9 Wendel U, Gonzales J, Hummel W. Neonatal screening for maple syrup urine disease by an enzyme-mediated colorimaple syrup urine disease by an enzyme-mediated

10 Naylor EW, Guthrie R. Newborn screening for maple syrup urine disease (branched-chain ketonuria). Pediatrics 1976;61:262-6.

11 Levy H. Hammersen G. Newborn screening for galactosaemia and other galactose metabolic defects. $\mathcal{F}$ Pediatr 1978;92:871-7.

12 Pang S, Hotchkiss J, Drash AL, et al. A microfilter paper method for 17-hydroxyprogesterone radioimmunoassay: its application for rapid screening for congenital adrenal hyperplasia. F Clin Endocrinol Metab 1977;45:1003-8.

13 Dove Pettit DA, Amador PS, Wolf B. The quantitation of biotinidase activity in dried blood spots using microtransfer plates: identification of biotinidase-deficient and heterozygous individuals. Anal Biochem 1989;179:371-4.

14 Bartlett K. Methods for the investigation of hypoglycaemia with particular reference to inherited disorders of mitochondrial $\beta$-oxidation. 7 Clin Endocrinol Metab 1993;7:643-67.

15 Eaton SJ, Bartlett K, Pourfarzam M. Mammalian mitochondrial $\beta$-oxidation. Biochem f 1996;320:345-57.

16 Chace DH, Millington DS, Terada N, Kahler SG, Roe CR, Hofman LF. Rapid diagnosis of phenylketonuria by quantitative analysis for phenylalanine and tyrosine in neonatal blood spots by tandem mass spectrometry. Clin Chem 1993;39:66-71.

17 Millington DS, Norwood DL, Kodo N, Roe CR, Inoue F. Application of fast atom bombardment with tandem mass spectrometry and liquid chromatography/mass spectrometry to the analysis of ascyl-carnitines in human urine, blood and tissue. Anal Biochem 1989;180:331-9.

18 Pourfarzam M, Besley GTN, Addison GM, Turnbull DM, Craft AW, Bartlett K. Tandem mass spectrometric analysis of dried blood spots is effective for the screening of multiple inherited metabolic disorders. Clin Sci (in press). 